\title{
Discrete search with multiple sensors
}

\author{
Nah-Oak Song ${ }^{1}$, Demosthenis Teneketzis ${ }^{2}$ \\ ${ }^{1} 100$ Bureau Dr. Stop 8920, Advanced Network Technologies Division, National Institute \\ of Standards and Technology, Gaithersburg, MD 20899-8920 \\ (e-mail: nsong@nist.gov) \\ 21301 Beal Avenue, Department of Electrical Engineering and Computer Science, \\ University of Michigan, Ann Arbor, MI 48109-2122 (e-mail: teneketzis@eecs.umich.edu)
}

Manuscript received: August 2003/Final version received: December 2003

\begin{abstract}
A stationary object is hidden in location $i, i=1,2, \cdots, K$, with probability $p_{i}$. There are $M$ sensors available and each location can be searched by at most one sensor at each instant of time. Each search of a location takes one unit of time and is conducted independently of previous searches, so that a search of location $i$ finds the object, if it is in that location, with probability $\alpha_{i}$. After each search of a location a sensor may either continue to search the same location or switch without any delay to another location. We determine optimal search strategies that maximize the total probability of successful search in $N$ units of time, discuss an implementation of an optimal search strategy, and specify conditions under which the solution can be obtained by a forward induction argument. Finally, we discuss the relationship to multi-armed bandits with multiple plays.
\end{abstract}

Key words: Search problem, Multiple sensors, Multi-armed bandits, Gittins index, Forward induction

\section{Introduction - problem formulation}

We formulate and investigate the following search problem with multiple sensors.

\section{Problem (P)}

A stationary object is hidden in location $i, i=1,2, \cdots, K$, with probability $p_{i}$, $\sum_{i=1}^{K} p_{i}=1$. There are $M(1<M<K)$ sensors available and each location can be searched by at most one sensor at each instant of time. Each search of a location takes one unit of time and is conducted independently of previous searches, so that a search of location $i, i=1,2, \cdots, K$, finds the object, given that it is in that location, with probability $\alpha_{i}, 0<\alpha_{i}<1$. After each search of 
a location a sensor may either continue to search the same location or switch without any delay to another location. The information about $p_{i}$ and $\alpha_{i}$ for each location $i, i=1,2, \cdots, K$, is given in advance. The objective is to find a search strategy that maximizes the total probability of successful search in $N$ units of time.

Problem (P) is a discrete search problem because the search space is discrete. Conventional discrete search problems (see [1], [6], [7], [10], [11], [15], [18], [20], [21]) are formulated as follows: A stationary object is hidden in one of $K$ locations. The probability that the object is in location $i$ is $p_{i}$. There is one sensor available. The probability that the object is found in the $j$-th search of location $i$ is $\alpha_{i j}$ if the object is in location $i$. The $j$-th search of location $i$ costs $c_{i j}$. When there is no time limitation, the objective is to find a search strategy that minimizes the expected cost until the object is found (see [1], [6], [7], [15], [18], [20], [23]). In the case where the maximum allowable time (or budget) for search is limited two objectives have been considered. The objective in whereabouts search is to find a search strategy that maximizes the probability of correctly stating the location that the object is hidden (see [1], [11], [20], [21]). In other search problems, the objective is to find a search strategy that maximizes the total probability of successful search (see [1], [10], [18], [20]).

Discrete search problems other than the conventional ones have been investigated in [1]-[5], [8], [12]-[14], [16], [18]-[20], [22]. In [12] Kelly investigated a discrete search problem where the object is always found if it is in the location that is being searched, that is, $\alpha_{i}=1$. In [13] Kelly considered a discrete search problem where a search of location $i, i=1,2, \cdots, K$, detects the object with probability $d$ given that the object is in location $i$, that is, $\alpha_{i}=d$, and this detection probability $d$ is fixed throughout the conduct of the search but is not known. Discrete search problems where a penalty (or delay) is incurred when the sensor switches locations were investigated in [1], [8], [14], [16], [24]. The discrete search problems when there are more than one stationary hidden objects have been investigated in [3], [4], [19]; search problems with a moving object have been investigated in [5], [22].

Discrete search problems investigated so far assume that there is only one sensor available for conducting search. All problems other than Arkin's (see [2]) assume that only one location is searched at each instant of time by the sensor. In [2] Arkin assumes that only one sensor is available but it can split its effort among any number of locations. The discrete search problem investigated by Arkin in [2] is different from Problem (P) in the following aspects: (i) The number of locations that are searched at each instant of time is time-varying; more specifically, according to Arkin's uniformly optimal strategy, the number of locations searched at each time instant is nondecreasing in time; (ii) The detection probability in any location (if the object is there) depends on the number of locations that are being searched and not on the location itself; (iii) Arkin's problem is formulated in continuous time.

To the best of our knowledge, Problem $(\mathbf{P})$ is the first investigation of a discrete search problem with multiple sensors.

The rest of paper is organized as follows. A solution to Problem (P) and an implementation of an optimal search strategy are presented in Section 2. Conditions under which the solution to Problem (P) can be obtained by forward induction are also presented in Section 2. The relation of 
Problem (P) to multi-armed bandits with multiple plays is discussed in Section 3. Conclusions and suggestions for further research appear in Section 4.

\section{Analysis}

Our analysis proceeds as follows: In Section 2.1 we present a preliminary result that allows us to restrict attention to a specific class of search strategies in order to determine a solution to Problem (P). We determine a class of optimal search strategies for Problem (P) in Section 2.2, and in Section 2.3 we discuss implementation of these strategies. In Section 2.4 we identify conditions under which the solution to Problem (P) can be obtained by forward induction.

\subsection{A Preliminary Result}

We define by $\pi_{i j}, i=1,2, \cdots, K, j=1,2, \cdots, N$, the probability that the object is found when the $j$ th search of location $i$ is conducted (see Table 1). Since we assume that each location can be searched by at most one sensor at each instant of time and $N$ time units are available, the probabilities, $\pi_{i j}$, $i=1,2, \cdots, K, j=N+1, N+2, \cdots$, are of no interest for Problem (P). The above defined probabilities have the following property:

Lemma 2.1. The probability $\pi_{i j}, i=1,2, \cdots, K, j=1,2, \cdots, N$, does not depend on the number of times other locations have been searched before the $j$ th search of location $i$ is conducted. That is, for $i=1,2, \cdots, K$, we have

$$
\pi_{i j}=p_{i} \alpha_{i}\left(1-\alpha_{i}\right)^{j-1}, \quad j=1,2, \cdots, N .
$$

\section{Proof. See Appendix A.}

As a result of Lemma 2.1 the total probability of successful search in $N$ units of time depends only on the number of times a strategy searches each location. Thus, without loss of optimality, we can restrict attention to search strategies of the form

$$
g(N):=\left(g_{1}, g_{2}, \cdots, g_{K}\right),
$$

where $g_{i}, i=1,2, \cdots, K$, denotes the number of searches of location $i$ according to $g(N)$ in $N$ units of time,

$$
0 \leq g_{i} \leq N,
$$

Table 1. Table of the probabilities $\pi_{i j}, i=1,2, \cdots, K, j=1,2, \cdots, N$.

\begin{tabular}{llll}
\hline Location 1 & Location 2 & $\cdots$ & Location K \\
\hline$\pi_{11}$ & $\pi_{21}$ & & $\pi_{K 1}$ \\
$\pi_{12}$ & $\pi_{22}$ & $\cdots$ & $\pi_{K 2}$ \\
$\pi_{13}$ & $\pi_{23}$ & $\vdots$ & $\pi_{K 3}$ \\
$\vdots$ & $\vdots$ & $\cdots$ & $\vdots$ \\
$\pi_{1 N}$ & $\pi_{2 N}$ & $\cdots$ & $\pi_{K N}$ \\
\hline
\end{tabular}




$$
\sum_{i=1}^{K} g_{i}=M N .
$$

Note that Eqs. (2) - (4) describe not a single strategy but a set of strategies. Each strategy in this set has the following feature: it visits location $i, i=1,2, \cdots, K, g_{i}$ times, possibly at different instances during the period $\{1,2, \cdots, N\}$.

\subsection{An Optimal Strategy for Problem (P)}

An optimal search strategy for Problem $(\mathbf{P})$ is characterized by the following result.

Theorem 2.1. Let $\mathcal{L}$ denote the set of $M N$ largest numbers $\pi_{i j}, i=1,2, \cdots, K$, $j=1,2, \cdots, N$, from Table 1 . There exists one search strategy $g^{*}(N)$ defined by

$$
\begin{aligned}
& g^{*}(N):=\left(g_{1}^{*}, g_{2}^{*}, \cdots, g_{K}^{*}\right) . \\
& g_{i}^{*}=\sum_{j=1}^{N} I\left(\pi_{i j}\right), \quad i=1,2, \cdots, K, \\
& I\left(\pi_{i j}\right)= \begin{cases}1, & \text { if } \pi_{i j} \in \mathcal{L}, \\
0, & \text { otherwise, }\end{cases}
\end{aligned}
$$

that is optimal for Problem (P).

Proof. Without any loss of optimality, restrict attention to search strategies $g(N)$, satisfying (2)-(4), and denote by

$$
P^{g(N)}:=\operatorname{Prob}(\text { object is found in N units of time under strategy } g(N)) \text {. }
$$

Then, from Lemma 2.1 it follows that for any $g(N)$ in the above class of strategies

$$
P^{g(N)}=\sum_{i=1}^{K} \sum_{j=1}^{g_{i}} \pi_{i j} .
$$

Therefore,

$$
P^{g^{*}(N)}=\sum_{i=1}^{K} \sum_{j=1}^{g_{i}^{*}} \pi_{i j},
$$

Consider any search strategy $g^{\prime}(N):=\left(g_{1}^{\prime}, g_{2}^{\prime}, \cdots, g_{K}^{\prime}\right)$ (in the above mentioned class of strategies) that is different from $g^{*}(N)$, that is, there are at least two distinct locations $i, j \in 1,2, \cdots, K$ such that $g_{i}^{*} \neq g_{i}^{\prime}, g_{j}^{*} \neq g_{j}^{\prime}$. Since

$$
P^{g^{\prime}(N)}=\sum_{i=1}^{K} \sum_{j=1}^{g_{i}^{\prime}} \pi_{i j}
$$


and (6)-(7) are true, it follows that

$$
P^{g^{*}(N)} \geq P^{g^{\prime}(N)} \text {. }
$$

The proof of the theorem will be complete if we can demonstrate that there exists at least one $g^{*}(N)$, described by (5) - (7), that is a feasible strategy. For that matter, we first note that because of Lemma 2.1 the probabilities $\pi_{i j}$, $i=1,2, \cdots, K$, are monotone decreasing in $j$; so, if $\pi_{i j^{\prime}}$ is in the set $\mathcal{L}$, all $\pi_{i j}$, $j<j^{\prime}$, are also in $\mathcal{L}$. Furthermore, in Section 2.3 we show that there is an implementation of $g^{*}(N)$ that satisfies the following constraint: at most one sensor is allocated to each location at each instant of time. Hence $g^{*}(N)$ is feasible.

\subsection{Implementation of an Optimal Search Strategy}

The result of Theorem 2.1 characterizes an optimal search strategy for Problem (P) but does not provide enough information about the implementation of such a strategy. This happens because the result of Theorem 2.1 does not explicitly specify how to deal with the requirement that at most one sensor can be allocated to each location at each instant of time. Indeed, a search strategy specified only by (5) - (7) is not necessarily feasible for Problem (P) as the following example shows.

Example 2.1. Consider Problem (P) with $K=3, N=3$ and two sensors. Suppose $g^{*}(3)=(2,2,2)$. The implementation of $g^{*}(3)$ that assigns the two sensors to locations 1 and 2 for the first two units of time is not feasible for Problem (P).

The following procedure leads to an implementation of an optimal search strategy $g^{*}(N):=\left(g_{1}^{*}, g_{2}^{*}, \cdots, g_{K}^{*}\right)$ that is described by Theorem 2.1 and is feasible for Problem (P).

An Implementation of an optimal search strategy $g^{*}(N)$. Let

$$
\begin{aligned}
& R(N):=\left(r_{1}^{N}, r_{2}^{N}, \cdots, r_{K}^{N}\right):=g^{*}(N), \\
& R(t)=\left(r_{1}^{t}, r_{2}^{t}, \cdots, r_{K}^{t}\right) .
\end{aligned}
$$

Define

$$
l(t):=M \text { largest numbers in } R(t) .
$$

Start at $t=N$; for $t=2,3, \cdots, N$, allocate a sensor to location $i$ if $r_{i}^{t} \in l(t)$ and set

$$
r_{i}^{t-1}= \begin{cases}r_{i}^{t}-1, & \text { if } r_{i}^{t} \in l(t) \\ r_{i}^{t}, & \text { otherwise. }\end{cases}
$$

At $t=1$ allocate the sensors to the $M$ locations with $r_{i}^{1}=1$.

The above procedure implements the search strategy $g^{*}(N)$.

Proof. It suffices to show that the above procedure leads to the following inequalities:

$$
r_{i}^{t} \leq t, \quad i=1,2, \cdots, K, \text { and } t=1,2, \cdots, N,
$$




$$
\sum_{i=1}^{K} I\left(r_{i}^{t}=t\right) \leq M, \quad t=2, \cdots, N
$$

and

$$
\sum_{i=1}^{K} I\left(r_{i}^{1}=1\right)=M
$$

where, for all $t=1,2, \cdots, N$,

$$
I\left(r_{i}^{t}=t\right)= \begin{cases}1, & \text { if } r_{i}^{t}=t, \\ 0, & \text { otherwise } .\end{cases}
$$

First, note that (17) and (18) are true for $t=N$ because of the formulation of Problem (P).

Assume that (17)-(18) are true for $t$; we want to prove that they are also true for $t-1, t>1$. The inequalities (17), (18) at $t$ imply that all $r_{i}^{t}=t$ are in the set $l(t)$. Consequently, because of (16) we get

$$
r_{i}^{t-1} \leq t-1
$$

for all $i=1,2, \cdots, K$. Furthermore,

$$
\sum_{i=1}^{K} I\left(r_{i}^{t-1}=t-1\right) \leq M,
$$

must hold, because if the opposite is true then

$$
\sum_{i=1}^{K} r_{i}^{N}=\sum_{i=1}^{K} g_{i}^{*}>M N
$$

must hold and (4) is violated. At $t=1$ we have, as a result of (21) and (22),

$$
r_{i}^{1} \leq 1
$$

for all $i=1,2, \cdots, K$, and

$$
\sum_{i=1}^{K} I\left(r_{i}^{1}=1\right) \leq M
$$

If

$$
\sum_{i=1}^{K} I\left(r_{i}^{1}=1\right)<M
$$

is true, then

$$
\sum_{i=1}^{K} r_{i}^{N}=\sum_{i=1}^{K} g_{i}^{*}<M N
$$

will hold and (4) is violated. Consequently, at $t=1$

$$
\sum_{i=1}^{K} I\left(r_{i}^{1}=1\right)=M \text {. }
$$


Therefore, the above described procedure implements an optimal search strategy, specified by Theorem 2.1; furthermore, it satisfies the requirement that at most one sensor can be allocated to each location at each instant of time.

In the above procedure, locations determined by $l(t), t=1,2, \cdots, N$, may not be uniquely defined. In such a case any choice of $M$ locations that correspond to the $M$ largest numbers in $R(t)$ will lead to an implementation of an optimal search strategy.

\subsection{Is Forward Induction Optimal for Problem $(P)$ ?}

Consider now the strategy $g^{I}$ that allocates, at each instant of time, the sensors to the locations with the $M$ highest probabilities of detection. A question that naturally arises at this point is the following. Is $g^{I}$ always an optimal search strategy for Problem (P)? The following example shows that $g^{I}$ is not always optimal. Denote by $g^{I}(N)$ the search strategy $g^{I}$ for the $N$-horizon problem ( $N$-fixed).

Example 2.2. Consider Problem (P) with $K=3, N=3$ and two sensors. Suppose

$$
\begin{aligned}
& p_{1}=0.3, \quad \alpha_{1}=0.3 \\
& p_{2}=0.5, \quad \alpha_{2}=0.15 \\
& p_{3}=0.2, \quad \alpha_{3}=0.4
\end{aligned}
$$

The probabilities $\pi_{i j}, i=1,2,3, j=1,2,3$, are given in Table 2 .

An optimal search strategy for this problem is given by

$$
g^{*}(3)=(2,3,1)
$$

and the corresponding probability that the object is found in three units of time under $g^{*}(3)$ is

$$
P^{g^{*}(3)}=0.4259375 \text {. }
$$

The search strategy $g^{I(3)}$ searches locations 1 and 3 at time 1 , locations 1 and 2 at time 2, and locations 2 and 3 at time 3. The probability that the object is found in 3 units of time under $g^{I(3)}$ is

$$
P^{g^{I}(3)}=0.41975 \text {. }
$$

Consequently, $g^{I}(3)$ is not an optimal search strategy.

Therefore, it is worthwhile investigating the conditions under which $g^{I}(N)$ is optimal.

We consider first Problem (P) with fixed finite horizon $N$. The following result provides a condition necessary and sufficient guarantee the optimality of $g^{I}(N)$ for the abovementioned problem.

Table 2. The probabilities $\pi_{i j}, i=1,2,3, j=1,2,3$ for Example 2.2.

\begin{tabular}{lll}
\hline Location 1 & Location 2 & Location 3 \\
\hline$\pi_{11}=0.09$ & $\pi_{21}=0.075$ & $\pi_{31}=0.08$ \\
$\pi_{12}=0.063$ & $\pi_{22}=0.06375$ & $\pi_{32}=0.048$ \\
$\pi_{13}=0.0441$ & $\pi_{23}=0.0541875$ & $\pi_{33}=0.0288$ \\
\hline
\end{tabular}


Theorem 2.2. The search strategy $g^{I}(N)$ is optimal for Problem (P) with fixed finite horizon $N$ iff the following condition $\mathbf{C 1}$ holds:

C1. $g^{I}(N)$ selects the $M N$ largest numbers among the probabilities $\pi_{i j}$, $i=1,2, \cdots, K, j=1,2, \cdots, N$.

Proof. According to Theorem 2.1, $g^{I}(N)$ will be an optimal search strategy for Problem (P) with (fixed) horizon $N$ iff it selects locations so that the corresponding probabilities of detection are the $M N$ largest numbers among $\pi_{i j}, i=1,2, \cdots, K, j=1,2, \cdots, N$.

Note that Condition $\mathbf{C 1}$ of Theorem 2.2 guarantees the optimality of the search strategy $g^{I}(N)$ for Problem $(\mathbf{P})$ with a fixed horizon $N$, but it does not necessarily guarantee that the truncation of $g^{I}(N)$ to the first $k$ steps $(k=1,2, \cdots, N-1)$, denoted by $g_{k}^{I}(N)$, gives an optimal search strategy for the $k$-horizon problem, as the following example shows:

Example 2.3. Consider Problem (P) with $K=3, N=3$ and two sensors. Suppose

$$
\begin{array}{cc}
p_{1}=0.3, & \alpha_{1}=0.3 \\
p_{2}=0.41, & \alpha_{2}=0.2 \\
p_{3}=0.29, & \alpha_{3}=0.3
\end{array}
$$

The probabilities $\pi_{i j}, i=1,2,3, j=1,2,3$, are given in Table 3 .

An optimal search strategy for this problem is given by

$g^{*}(3)=(2,2,2)$.

The search strategy $g^{I}(3)$ searches locations 1 and 3 at time 1 , locations 1 and 2 at time 2, and locations 2 and 3 at time 3. Hence,

$$
g^{I}(3)=(2,2,2),
$$

therefore, $g^{I}(3)$ is optimal. However, the truncation of $g^{I}(3)$ to the first two steps, is

$$
g_{2}^{I}(3)=(2,1,1)
$$

while an optimal search strategy for the 2-horizon problem, according to Theorem 2.1, is

$$
g^{*}(2)=(1,2,1)
$$

Consequently, Condition $\mathbf{C 1}$ does not guarantee the optimality of $g^{I}$ for Problem (P) with any horizon $N$.

Next we investigate conditions under which the search strategy $g^{I}$ is optimal for Problem (P) with any horizon $N, N=1,2, \cdots$. If such conditions

Table 3. The probabilities $\pi_{i j}, i=1,2,3, j=1,2,3$, for Example 2.3.

\begin{tabular}{lll}
\hline Location 1 & Location 2 & Location 3 \\
\hline$\pi_{11}=0.09$ & $\pi_{21}=0.082$ & $\pi_{31}=0.087$ \\
$\pi_{12}=0.063$ & $\pi_{22}=0.0656$ & $\pi_{32}=0.0609$ \\
$\pi_{13}=0.0441$ & $\pi_{23}=0.05248$ & $\pi_{33}=0.04263$ \\
\hline
\end{tabular}


exist an optimal search strategy for Problem $(\mathbf{P})$ can be obtained by forward induction.

The following result provides a condition necessary and sufficient to guarantee the optimality of the search strategy $g^{I}$ for Problem $(\mathbf{P})$ with any horizon $N$.

Theorem 2.3. The search strategy $g^{I}$ is optimal for Problem (P) with any horizon $N$ iff the following condition $\mathbf{C 2}$ holds:

C2. Consider any time $t, t=1,2, \cdots$, and let $g_{i(t-1)}$ denote the number of times location $i, i=1,2, \cdots, K$, has been searched up until time $t-1$ (including time $t-1)$ according to $g^{I}$. Consider the $M$ largest numbers among $\pi_{i j_{i}(t)}, i=1,2, \cdots, K, j_{i}(t)=g_{i(t-1)}+1$. These numbers are also the $M$ largest numbers among $\pi_{i j_{i}}, i=1,2, \cdots, K, j_{i}=g_{i(t-1)}+1, \cdots, t$.

Proof. Sufficiency: Suppose Condition C2 holds. This condition ensures that for every $t, t=1,2, \cdots$, the search strategy $g^{I}(t)$ selects the $M t$ largest numbers among $\pi_{i j}, i=1,2, \cdots, K, j=1,2, \cdots, t$. Hence according to Theorem 2.1, for every $t, t=1,2, \cdots$, the search strategy $g^{I}(t)$ is optimal for Problem $(\mathbf{P})$.

Necessity: Suppose the search strategy $g^{I}$ is optimal for Problem $(\mathbf{P})$ for all $t, t=1,2, \cdots$, but Condition $\mathbf{C 2}$ is not satisfied for all $t, t=1,2, \cdots$. Let $s$ be the first instant Condition C2 is not satisfied. Then $g^{I}(s)$ does not select the largest $M s$ numbers among $\pi_{i j}, i=1,2, \cdots, K, j=1,2, \cdots, s$. If $g^{*}(s)$ is an optimal search strategy for Problem (P) satisfying the conditions of Theorem 2.1, then, because of (8) and (9)

$$
P^{g^{*}(s)}>P^{g^{I}(s)}
$$

and this contradicts the optimality of the search strategy $g^{I}$ for all $t$.

The results of Theorems 2.2 and 2.3 show that under certain conditions the solution of Problem (P) $g^{I}$ is of an "index type." This is further explained in the next section where the relationship between Problem $(\mathbf{P})$ and multiarmed bandits with multiple plays is explored.

\section{Relation of Problem (P) to Multi-Armed Bandits with Multiple Plays}

Problem (P) can be viewed as a finite-horizon deterministic multi-armed bandit with multiple plays and discount factor equal to one (see [9], [17], [18]) as follows. Each location is associated with an arm of a multi-armed bandit; thus, there are $K$ arms. At time $t, t=1,2, \cdots, N$, the state of the $i$-th arm, $i=1,2, \cdots, K$, is

$$
x_{i}(t)=g_{i(t-1)},
$$

where $g_{i(t-1)}$ denotes the number of times arm $i$ has been played up until time $t-1$, including time $t-1$ (i.e. $g_{i(t-1)}$ denotes the number of times location $i$ has been searched up until time $t-1)$. $M$ arms $(M>1)$ are played at each instant of time. If arm $i$ is played at time $t$ a reward

$$
R\left(x_{i}(t)\right)=p_{i} \alpha_{i}\left(1-\alpha_{i}\right)^{x_{i}(t)}
$$


is obtained and the arm moves to state

$$
x_{i}(t+1)=x_{i}(t)+1=g_{i(t-1)}+1 \text {. }
$$

If arm $i$ is not played at time $t$, no reward is obtained from this arm and the arm's state remains "frozen", i.e.

$$
x_{i}(t+1)=x_{i}(t)=g_{i(t-1)} \text {. }
$$

The objective is to determine an allocation policy to maximize the reward

$$
R=\sum_{t=1}^{N} \sum_{i \in k(t)} R\left(x_{i}(t)\right),
$$

where $k(t)$ denote the set of $M$ arms being played at time $t$.

The Gittins index [9] for each arm is achieved at time 1, because, for all arms, the numbers

$$
\pi_{i j}=p_{i} \alpha_{i}\left(1-\alpha_{i}\right)^{j-1}, \quad j=1,2, \cdots,
$$

which give the sequence of rewards obtained from playing arm $i$, $i=1,2, \cdots, K$, form a strictly decreasing sequence in $j$.

For the above problem, define the Gittins index rule to be the allocation policy according to which the arms with the $M$ highest indices are being played at each instant of time. Thus, the Gittins index rule coincides with the search strategy $g^{I}$. Consequently, the following results hold:

Theorem 3.1. Under Condition $\mathbf{C 1}$ of Theorem 2.2, the Gittins index rule is optimal for Problem (P) with fixed finite horizon $N$.

Theorem 3.2. Under Condition $\mathbf{C 2}$ of Theorem 2.3, the Gittins index rule is optimal for Problem (P)with any horizon $N$.

Remark. It is known that the Gittins index rule is, in general, not optimal for the multi-armed bandit problem with multiple plays [17]. The results in [17] provide conditions sufficient to guarantee the optimality of the Gittins index rule for the general multi-armed bandit problem with multiple plays and discount factor strictly less than one. The results here (Theorem 3.2) are for the undiscounted problem and are particularly simple because the index is always achieved at time 1 for all arms.

\section{Conclusion}

We formulated a search problem with multiple sensors. We determined optimal search strategies for this problem, discussed an implementation of an optimal search strategy, and specified conditions under which an optimal search strategy can be obtained by forward induction.

A critical assumption in the problem formulated in this paper is that the sensors can move instantaneously from one location to another. In reality, when a sensor switches between different locations a delay is incurred. Thus, search problems with switching delays are worth investigating. The inclusion of switching delays drastically changes the nature of search problems. The resulting problems are considerably more difficult than the corresponding 
ones without switching delays. To the best of our knowledge, there are no analytical results for search problems with multiple sensors and switching delays presently available.

Acknowledgements. This research was supported in part by NSF Grant NCR-9204419, AFOSR Grant F49620-96-1-0028, ARO Grant DAAH04-96-1-0377 and ONR Grant N00014-03-1-0232. Nah-Oak Song's work was also supported in part by a Barbour Scholarship from the University of Michigan

\section{Appendix A}

Proof of Lemma 2.1. For $i=1,2, \cdots, K, j=1,2, \cdots, N, t=1,2, \cdots, N$, define the following events:

$A(i, j):=$ the object is found when the $j$ th search of location $i$ is conducted, $A^{c}(i, j):=$ the object is not found when the $j$ th search of location $i$ is conducted,

$T_{i j}:=$ the time that the $j$ th search of location $i$ is conducted,

$O(i):=$ the object is in location $i$,

$S(i, t):=$ the object is found when location $i$ is searched at time $t$,

$S^{c}(i, t):=$ the object is not found when location $i$ is searched at time $t$,

$U_{i}\left(t_{1}, t_{2}\right):=$ unsuccessful search of locations other than location $i$ during time

interval $\left[t_{1}, t_{2}\right)$. For any $t_{1}<t_{2}$ the time interval $\left[t_{1}, t_{2}\right)$ is, by definition, the set of times $\left\{t_{1}, t_{1}+1, \cdots, t_{2}-2, t_{2}-1\right\}$.

Then, we have

$$
\begin{aligned}
\pi_{i 1} & =P[A(i, 1)] \\
& =\sum_{t=1}^{N} P\left[A(i, 1) \mid T_{i 1}=t\right] P\left[T_{i 1}=t\right] \\
& =\sum_{t=1}^{N} P\left[O(i), S(i, t), U_{i}(1, t)\right] P\left[T_{i 1}=t\right] \\
& =\sum_{t=1}^{N} P\left[S(i, t) \mid O(i), U_{i}(1, t)\right] P\left[O(i), U_{i}(1, t)\right] P\left[T_{i 1}=t\right] .
\end{aligned}
$$

Since each search is conducted independently

$$
P\left[S(i, t) \mid O(i), U_{i}(1, t)\right]=\alpha_{i},
$$

and furthermore,

$$
\begin{array}{r}
P\left[O(i), U_{i}(1, t)\right]=P[O(i)] \\
=p_{i} .
\end{array}
$$

Because of (A.2) and (A.3), (A.1) gives

$$
\pi_{i 1}=p_{i} \alpha_{i} \sum_{t=1}^{N} P\left[T_{i 1}=t\right]=p_{i} \alpha_{i} .
$$


In general, for $j=2,3, \cdots, N$, we have

$$
\begin{aligned}
\pi_{i j}= & P[A(i, j)] \\
= & \sum_{\substack{t_{j}=\\
t_{j-1}+1}}^{N} \sum_{t_{j-1}=}^{N-1} \cdots \sum_{t_{j-2}=1}^{N-j+1} P\left[A(i, j), A^{c}(i, j-1), \cdots, A^{c}(i, 1) \mid T_{i 1}=t_{1},\right. \\
& \left.\cdots, T_{i(j-1)}=t_{j-1}, T_{i j}=t_{j}\right] P\left[T_{i 1}=t_{1}, \cdots, T_{i(j-1)}=t_{j-1}, T_{i j}=t_{j}\right] \\
= & \sum_{\substack{t_{j}=\\
t_{j-1}+1}}^{N} \sum_{t_{j-1}=}^{N-1} \cdots \sum_{t_{j-2}+1}^{N-j+1} P\left[O(i), S\left(i, t_{j}\right), S^{c}\left(i, t_{j-1}\right), \cdots, S^{c}\left(i, t_{1}\right),\right. \\
& \left.U_{i}\left(1, t_{1}\right), \cdots, U_{i}\left(t_{j-2}, t_{j-1}\right), U_{i}\left(t_{j-1}, t_{j}\right)\right] P\left[T_{i 1}=t_{1}, \cdots,\right. \\
& \left.T_{i(j-1)}=t_{j-1}, T_{i j}=t_{j}\right] \\
= & \sum_{t_{j}=}^{N} \sum_{t_{j-1}=}^{N-1} \cdots \sum_{t_{1}=1}^{N-j+1} P\left[S\left(i, t_{j}\right) \mid O(i), S^{c}\left(i, t_{j-1}\right), \cdots, S^{c}\left(i, t_{1}\right),\right. \\
& \left.U_{i}\left(1, t_{1}\right), \cdots, U_{i}\left(t_{j-2}, t_{j-1}\right), U_{i}\left(t_{j-1}, t_{j}\right)\right] P\left[S^{c}\left(i, t_{j-1}\right) \mid O(i),\right. \\
& \left.S^{c}\left(i, t_{j-2}\right), \cdots, S^{c}\left(i, t_{1}\right), U_{i}\left(1, t_{1}\right), \cdots, U_{i}\left(t_{j-2}, t_{j-1}\right), U_{i}\left(t_{j-1}, t_{j}\right)\right] \\
& \cdots P\left[S^{c}\left(i, t_{1}\right) \mid O(i), U_{i}\left(1, t_{1}\right), \cdots, U_{i}\left(t_{j-2}, t_{j-1}\right), U_{i}\left(t_{j-1}, t_{j}\right)\right] \\
& P\left[O(i), U_{i}\left(1, t_{1}\right), \cdots, U_{i}\left(t_{j-2}, t_{j-1}\right), U_{i}\left(t_{j-1}, t_{j}\right)\right] P\left[T_{i 1}=t_{1}, \cdots,\right. \\
& \left.T_{i(j-1)}=t_{j-1}, T_{i j}=t_{j}\right] .
\end{aligned}
$$

Since each search is conducted independently

$$
\begin{aligned}
& P\left[S\left(i, t_{j}\right) \mid O(i), S^{c}\left(i, t_{j-1}\right), \cdots, S^{c}\left(i, t_{1}\right), U_{i}\left(1, t_{1}\right),\right. \\
& \left.U_{i}\left(t_{j-2}, t_{j-1}\right), U_{i}\left(t_{j-1}, t_{j}\right)\right]=\alpha_{i}, \\
& P\left[S^{c}\left(i, t_{j^{\prime}}\right) \mid O(i), S^{c}\left(i, t_{j-2}\right), \cdots, S^{c}\left(i, t_{1}\right), U_{i}\left(1, t_{1}\right), \cdots, U_{i}\left(t_{j^{\prime}-1}, t_{j-1}\right),\right. \\
& \left.U_{i}\left(t_{j-1}, t_{j}\right)\right]=1-\alpha_{i}, j^{\prime}=1, \cdots, j-1,
\end{aligned}
$$

and furthermore,

$$
\begin{aligned}
P\left[O(i), U_{i}\left(1, t_{1}\right), \cdots, U_{i}\left(t_{j-2}, t_{j-1}\right), U_{i}\left(t_{j-1}, t_{j}\right)\right] & =P[O(i)] \\
& =p_{i} .
\end{aligned}
$$

Because of (A.6) - (A.8), (A.5) gives

$$
\begin{aligned}
\pi_{i j}= & p_{i} \alpha_{i}\left(1-\alpha_{i}\right)^{j-1} \sum_{\substack{t_{j}=\\
t_{j-1}+1}}^{N} \sum_{\substack{t_{j-1}=1 \\
t_{j-2}+1}}^{N-1} \cdots \sum_{t_{1}=1}^{N-j+1} \\
& P\left[T_{i 1}=t_{1}, \cdots, T_{i(j-1)}=t_{j-1}, T_{i j}=t_{j}\right] \\
= & p_{i} \alpha_{i}\left(1-\alpha_{i}\right)^{j-1} .
\end{aligned}
$$




\section{References}

[1] Ahlswede R, Wegener I (1987) Search problems. John Wiley \& Sons, Chichester

[2] Arkin VI (1964) A problem of optimum distribution of search effort. Theory Prob. Applications 9:159-160 (English translation)

[3] Assaf D, Zamir S (1985) Optimal sequential search: a Bayesian approach. Ann. Statist. 3:1213-1221

[4] Assaf D, Zamir S (1987) Continuous and discrete search for one of many objects. Operations Research Letters 6:205-209

[5] Assaf D, Sharlin A (1994) Dynamic search for a moving target. J. Appl. Prob. 31:438-457

[6] Black WL (1965) Discrete sequential search. Information and control 8:159-162

[7] Chew MC (1967) A sequential search procedure. Ann. Math. Statist. 38:494-502

[8] Gilbert EN (1959) Optimal search strategy. SIAM 7:413-424

[9] Gittins JC (1989) Multi-armed bandit allocation indices. John Wiley \& Sons, Chichester

[10] Kadane JB (1968) Discrete Search and the Neyman-Pearson Lemma. J. Math. Anal. and Appl. 22:156-171

[11] Kadane JB (1971) Optimal whereabouts search. Operations Research 19:894-904

[12] Kelly FP (1982) A remark on search and sequencing problems. Math. Oper. Res 88:422-432

[13] Kelly FP (1982) On optimal search with unknown detection probabilities. J. Math. Anal. and Appl. 88:422-432

[14] Lössner U, Wegener I (1982) Discrete sequential search with positive switch cost. Math. Oper. Res. 7:426-440

[15] Matula D (1964) A periodic optimal search. Amer. Math. Monthly 71:15-21

[16] Onaga K (1971) Optimal search for detecting a hidden object. SIAM 20:298-318

[17] Pandelis DG, Teneketzis D (1999) On the optimality of the Gittins index rule for multiarmed bandits with multiple plays. Math. Meth. Oper. Res. 50:449-461

[18] Ross SM (1983) Introduction to stochastic dynamic programming. Academic Press, Sandiego

[19] Sharlin A (1987) Optimal search for one of many objects hidden in two boxes. European J. Oper. Res. 6:205-209

[20] Stone LD (1975) Theory of optimal search. Academic Press, New York

[21] Tognetti KP (1968) An optimal strategy for a whereabouts search. Operations Research 16:209-211

[22] Webber RR (1986) Optimal search for a randomly moving object. J. Appl. Prob. 23:708-717

[23] Wegener I (1980) The discrete sequential search problem with nonrandom cost and overlook probabilities. Math. Oper. Res. 5:373-380

[24] Wegener I (1985) Optimal search with positive switch cost is NP-hard. Information Processing Letters 21:49-52 